\title{
Inhibitory Effects of Azelnidipine Tablets on Morning Hypertension
}

\author{
Kazuomi Kario $\cdot$ Yuki Sato $\cdot$ Masayuki Shirayama $\cdot$ \\ Megumi Takahashi - Kazuhito Shiosakai - Katsutoshi Hiramatsu • \\ Masahiro Komiya $\cdot$ Kazuyuki Shimada
}

Published online: 20 March 2013

(C) The Author(s) 2013. This article is published with open access at Springerlink.com

\begin{abstract}
Background Morning hypertension is a risk factor for cardiovascular and cerebrovascular events. Furthermore, it is a useful measure for definitive diagnosis of hypertension, and patients who self-assess their own blood pressure (BP) in the morning tend to exhibit better compliance with antihypertensive medication than those who do not.

Objective The objective of this analysis was to determine the BP- and pulse rate-lowering effects of azelnidipine, a long-acting dihydropyridine calcium antagonist administered once daily in the morning.

Methods We conducted the Azelnidipine Treatment for Hypertension Open-label Monitoring in the Early morning (At-HOME) Study by surveying patients who were taking azelnidipine. According to the study protocol, high systolic BP (SBP) was defined as $\geq 135 \mathrm{mmHg}$ when measured at home in the morning and $\geq 140 \mathrm{mmHg}$ when measured at the clinic during the day. A total of 5,433 hypertensive patients, who were registered at 1,011 medical institutions
\end{abstract}

Electronic supplementary material The online version of this article (doi:10.1007/s40268-013-0006-8) contains supplementary material, which is available to authorized users.

K. Kario $(\bowtie) \cdot$ K. Shimada

Division of Cardiovascular Medicine, Department of Medicine, Jichi Medical University School of Medicine, 3311-1 Yakushiji,

Shimotsuke, Tochigi 329-0498, Japan

e-mail: kkario@jichi.ac.jp

Y. Sato · M. Shirayama $\cdot$ M. Takahashi $\cdot$ K. Hiramatsu ·

M. Komiya

Post Marketing Study Management Department,

Daiichi Sankyo Co., Ltd, Tokyo, Japan

K. Shiosakai

Department of Data Science, Daiichi Sankyo Co., Ltd,

Tokyo, Japan across Japan, were enrolled in the study. Data obtained from 4,852 of these patients (mean age, 64.8 years; female, $52.9 \%$; previous medication with other antihypertensive agents used concomitantly with the present study agent, $45.5 \%$ ) were used for efficacy analysis.

Results At baseline, the subjects' mean [ \pm standard deviation] SBP/diastolic BP values at home in the morning, at the clinic during the day, and at home in the evening were $156.9 \pm 16.4 / 89.7 \pm 12.0,157.5 \pm 18.7 / 89.1 \pm 13.3$, and $150.2 \pm 17.6 / 85.6 \pm 12.2 \mathrm{mmHg}$, respectively. The mean pulse rates were $72.7 \pm 10.7,74.9 \pm 11.2$, and $72.5 \pm$ 9.6 beats/min, respectively. Patients whose BP was defined as high accounted for $83.4 \%$ of the study population, whereas $9.9 \%$ had 'masked' hypertension, defined as SBP of $\geq 135 \mathrm{mmHg}$ at home in the morning and $<140 \mathrm{mmHg}$ at the clinic. However, from 4 weeks after initiation of azelnidipine treatment till the end of the study at week 16 , all three daily BP determinations were significantly $(p<0.0001)$ lowered, and pulse rates at home in the morning, at the clinic, and at home in the evening were similarly and significantly reduced (by $-3.7 \pm 8.0,-3.5 \pm 9.5$, and $-3.5 \pm 7.3$ beats $/ \mathrm{min}$, respectively). Whereas achievement of home SBP of $<135$ $\mathrm{mmHg}$ in the morning was noted in only $6.6 \%$ of patients before the start of azelnidipine treatment, this was noted in $43.3 \%$ after 16 weeks. Meanwhile, achievement of clinic SBP of $<140 \mathrm{mmHg}$ was increased from $12.9 \%$ of patients to $56.1 \%$ of patients at the same timepoints. After azelnidipine treatment, $32.2 \%$ of patients had well-controlled hypertension in both the home and clinic settings. Adverse drug reactions occurred in $2.92 \%$ of patients $(154 / 5,265)$. All adverse drug reactions were as expected for the calcium antagonist class of agents.

Conclusion These data suggest that azelnidipine controlled morning hypertension well. Furthermore, azelnidipine reduced pulse rates significantly. 


\section{Introduction}

Morning hypertension and morning blood pressure (BP) surge are serious risk factors affecting cerebrovascular and cardiovascular events, and controlling them is expected to greatly improve the prognosis of patients with hypertension [1]. It was reported in the Jichi Morning-Hypertension Research (J-MORE) Pilot Study (performed in patients treated with antihypertensive drugs in Japan) that more than half of the patients who had well-controlled BP when it was measured at the clinic during the day (clinic BP) suffered from morning hypertension, and their BP measured at home in the morning (morning home BP) was poorly controlled [2]. Pickering et al. [3] compared normotension with masked hypertension and warned that the latter would increase the relative risk of cardiovascular events to an extent comparable with or higher than that of sustained hypertension. An epidemiological study performed in residents of Ohasama Machi in Iwate Prefecture, Japan, also found that morning home BP was a better predictor of cardiovascular disease or death than clinic BP [4], suggesting that measurement and control of morning home BP is very important for effective antihypertensive therapy. Measurement of BP at home is also useful for achieving better treatment compliance and for evaluating the effectiveness of antihypertensive drugs, and morning measurement before intake of medication, in particular, has been reported to be useful for the evaluation of sustained BP-lowering effects of antihypertensive drugs administered once daily [5]. Thus, more significant clinical findings from evaluation of antihypertensive drug efficacy would be expected using morning home $\mathrm{BP}$ as an index rather than using clinic BP.

Azelnidipine is a dihydropyridine calcium antagonist, which was synthesized by Ube Industries, Ltd. and developed by Sankyo Co., Ltd. (now known as Daiichi Sankyo Co., Ltd., Tokyo, Japan). This agent has a potent and sustained BP-lowering effect in various animal models of hypertension [6]. It has also been confirmed to have renoprotective effects (such as reducing proteinuria by dilating efferent arterioles), as well as cardioprotective, insulin resistance-improving, cerebroprotective, and anti-atherosclerotic effects $[7,8]$. In a comparative clinical study using the index of 24-h ambulatory BP monitoring, azelnidipine (with lipophilicity 17-fold higher than that of amlodipine) showed a sustained 24-h BP-lowering effect comparable to that of amlodipine [9]. Meanwhile, azelnidipine had no effect on pulse rates, unlike amlodipine, and had a mildly suppressive effect rather than an inductive effect on reflective tachycardia in response to hypotension [10]. These properties are thought to arise because azelnidipine hardly activates the sympathetic nervous system.
We investigated the suppressive effect of azelnidipine on BP measured at the clinic and at home, morning hypertension, and pulse rates, using data from the Azelnidipine Treatment for Hypertension Open-label Monitoring in the Early morning (At-HOME) Study, which was carried out as a special survey for post-marketing surveillance in daily clinical settings.

\section{Subjects and Methods}

\subsection{Subjects}

This study was conducted according to Article 14-4 (reexamination) of the Pharmaceutical Affairs Act, Japan, and in compliance with Good Post-marketing Study Practice (GPSP). For a list of participating centers [in Japanese], see the electronic supplementary material. The study included patients who met all of the following requirements at baseline when they started taking the study drug, azelnidipine $\left(\right.$ Calblock $^{\circledR}$ tablets; Daiichi Sankyo Co., Ltd.): (i) outpatient with hypertension; (ii) no previous use of the study drug; (iii) clinic BP measurement within 28 days prior to baseline; and (iv) morning home BP measurement using an electronic brachial-cuff device at least two times on separate dates within 28 days prior to baseline. The study was conducted using the central enrollment method, in which patients from contracted medical institutions nationwide were registered by the enrollment center within 14 days after the baseline date. The enrollment period was one year from May 2006, and the planned number of cases to be investigated was 5,000 .

The study drug was administered at the investigator's discretion, according to the dosage and administration instructions in the package insert, with no limit set on dose increases or decreases, or on pretreatment or concomitant use of antihypertensive drugs. The standard observation period was 16 weeks, during which the study drug was administered, except in cases of withdrawal or dropout.

\subsection{Outcome Measures}

We investigated the patient characteristics, study drug dosage, study drug compliance, pretreatment with antihypertensive drugs, use of concomitant drugs, clinical course, clinical examinations, conditions of BP measurement at home, and adverse events occurring during or after treatment with the study drug. In order to investigate the variables under actual conditions, the method of BP measurement and the timing of dosing and BP measurement during the observation period were not specified in the study protocol, and these decisions were left to the 
investigators. Investigators assessed safety on the basis of the results of patient interviews and clinical examinations.

\subsection{Subject Inclusion in Analysis Sets}

The following enrolled patients were excluded from the safety analysis population (Fig. 1): (i) those who did not return to the clinic after the initial visit, precluding assessment of adverse events; (ii) those who took no study drug; (iii) those with no written description of adverse events; and (iv) those who exceeded the timeframe for registration (ineligibility proven after data collection). From among the safety analysis population, the following patients were excluded from the efficacy analysis population: (i) those who were not outpatients with hypertension at baseline; (ii) those who had previously used the study drug; (iii) those with no clinic BP measurement within 28 days prior to the baseline date; (iv) those with no morning home BP measurement using an electronic brachial-cuff device within 28 days prior to the baseline date; and (v) those whose reported compliance was "[I] almost never take the study drug". Although at least two morning home BP measurements on separate dates were required for enrollment in the study, patients with only one morning home BP measurement were also included in the study analyses. It was confirmed that there were no major differences in the results of the primary analysis when only those patients with two measurements of BP (protocolcompliant cases) were included.

\subsection{Methods of Analysis}

A paired $t$-test was used to analyze changes in SBP, diastolic BP (DBP), and pulse rates between baseline and the endpoint of the investigation. Dunnett's test was performed to compare values at weeks $4,8,12$, and 16 with those at baseline. The tests were two-sided, with the significance level being set at $p=0.05$. Values were expressed as means \pm standard deviations (SDs). Changes in patient classification before and after azelnidipine administration were tabulated using clinic SBP of $\geq 140 \mathrm{mmHg}$ and morning home SBP of $\geq 135 \mathrm{mmHg}$ as indexes of

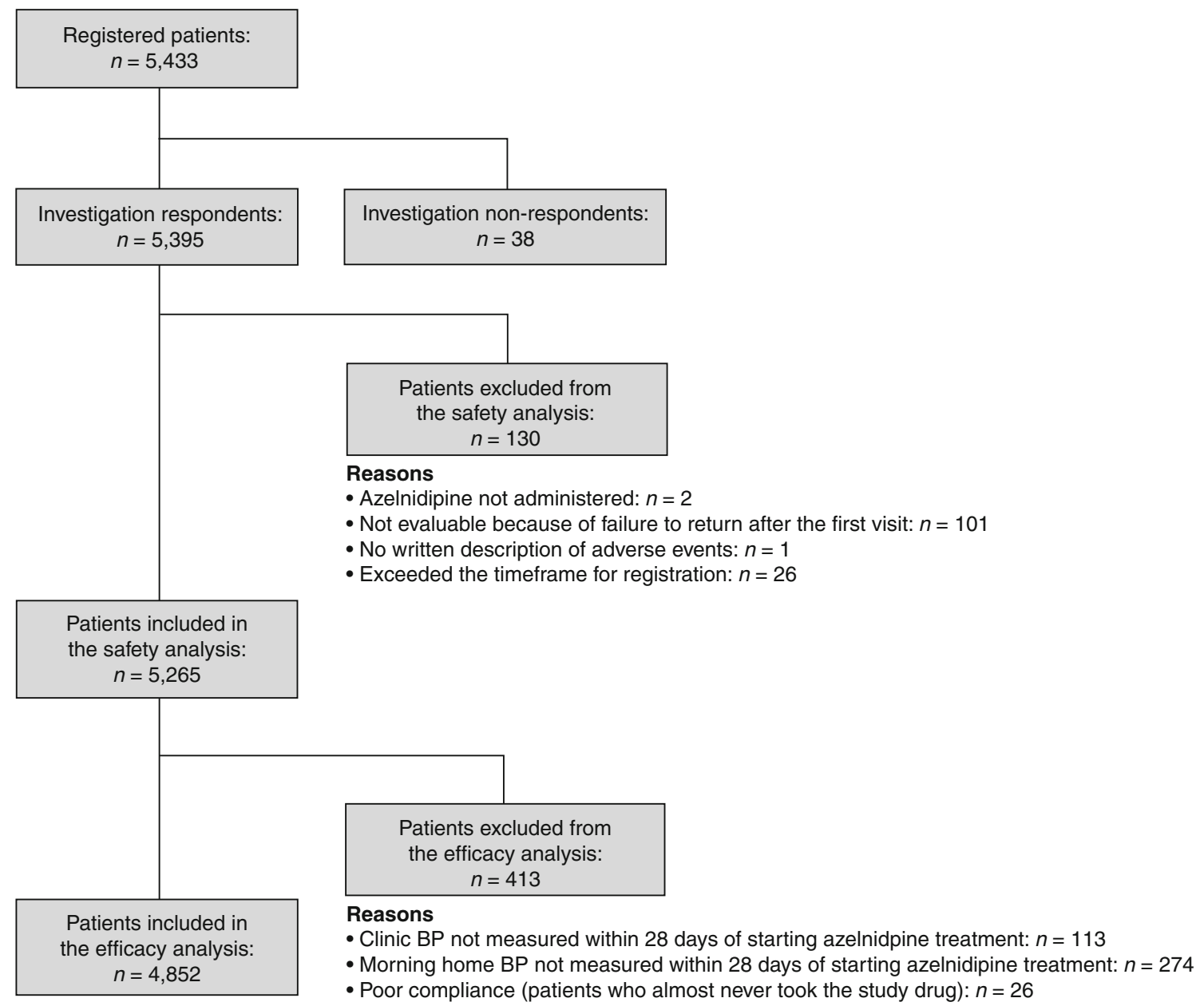

Fig. 1 Patient disposition in the current study. $B P$ blood pressure 
hypertension to classify hypertension as well controlled (clinic SBP of $<140 \mathrm{mmHg}$, morning home SBP of $<135 \mathrm{mmHg}$ ); white coat (clinic $\mathrm{SBP}$ of $\geq 140 \mathrm{mmHg}$, morning home SBP of $<135 \mathrm{mmHg}$ ); masked (clinic SBP of $<140 \mathrm{mmHg}$, morning home SBP of $\geq 135 \mathrm{mmHg}$ ); or poorly controlled (clinic SBP of $\geq 140 \mathrm{mmHg}$, morning home SBP of $\geq 135 \mathrm{mmHg}$ ). The McNemar test was used for evaluating changes in patient distribution by BP classification according to clinic SBP and morning home SBP before and after administration of azelnidipine.

Adverse events and adverse drug reactions were coded using the Medical Dictionary for Regulatory Activities (MedDRA)/J version 11.0 and classified according to their Preferred Terms.

\section{Results}

\subsection{Patient Disposition}

Figure 1 shows the patient disposition. A total of 5,433 patients from 1,011 medical institutions across Japan were registered. Safety analyses were performed in 5,265 patients after exclusion of 130 patients from investigation respondents, and efficacy analyses were performed in 4,852 patients after exclusion of 413 patients from safety analysis (Fig. 1).

\subsection{Patient Characteristics}

Table 1 shows the patient characteristics at baseline. The mean age was $64.8 \pm 11.9$ years, and $52.9 \%$ of patients were female. Hyperlipidemia was the most frequently observed complication, followed by diabetes mellitus and heart disease. The mean baseline SBP/DBP values were $157.5 \pm 18.7 /$ $89.1 \pm 13.3 \mathrm{mmHg}$ at the clinic, $156.9 \pm 16.4 / 89.7 \pm$ $12.0 \mathrm{mmHg}$ at home in the morning, and $150.2 \pm 17.6 /$ $85.6 \pm 12.2 \mathrm{mmHg}$ at home in the evening (evening home BP). The mean pulse rates were $74.9 \pm 11.2$ beats $/ \mathrm{min}$ (clinic), $72.7 \pm 10.7$ beats/min (morning home), and $72.5 \pm$ 9.6 beats/min (evening home). The proportion of poorly controlled hypertension, which was defined by both high clinic SBP and high morning home SBP, was $83.4 \%$, and the proportion of masked hypertension, which was defined by normal clinic SBP and high morning home SBP, was $9.9 \%$. During the observation period, morning home SBP was usually measured before breakfast and before dosing in a large proportion (85.2\%) of cases.

\subsection{Dosage of the Study Drug}

Table 2 shows the dosage of the study drug. Nearly $70 \%$ of patients received $16 \mathrm{mg}$ (the most frequent initial daily
Table 1 Patient characteristics at baseline $(n=4,852)$

\begin{tabular}{|c|c|}
\hline Characteristic & Value \\
\hline \multicolumn{2}{|l|}{ Gender $(n[\%])$} \\
\hline Male & $2,283[47.1]$ \\
\hline Female & $2,569[52.9]$ \\
\hline Age (years \pm SD) & $64.8 \pm 11.9$ \\
\hline$<15$ years $(n[\%])$ & $0[0.0]$ \\
\hline 15 to $<65$ years $(n[\%])$ & $2,239[46.1]$ \\
\hline 65 to $<75$ years $(n[\%])$ & $1,544[31.8]$ \\
\hline$\geq 75$ years $(n[\%])$ & $1,060[21.8]$ \\
\hline Not specified $(n[\%])$ & $9[0.2]$ \\
\hline $\mathrm{BMI}\left(\mathrm{kg} / \mathrm{m}^{2} \pm \mathrm{SD}\right)$ & $24.28 \pm 3.64$ \\
\hline$<18.5 \mathrm{~kg} / \mathrm{m}^{2}(n[\%])$ & $122[2.5]$ \\
\hline 18.5 to $<25 \mathrm{~kg} / \mathrm{m}^{2}$ ( $\left.n[\%]\right)$ & $1,992[41.1]$ \\
\hline$\geq 25 \mathrm{~kg} / \mathrm{m}^{2}(n[\%])$ & $1,305[26.9]$ \\
\hline Not calculable ( $n[\%])$ & $1,433[29.5]$ \\
\hline \multicolumn{2}{|l|}{ Diagnosis $(n[\%])$} \\
\hline Essential hypertension & $4,813[99.2]$ \\
\hline Other hypertension & $39[0.8]$ \\
\hline \multicolumn{2}{|l|}{$\mathrm{BP}$ and pulse rates } \\
\hline Clinic SBP $(\mathrm{mmHg} \pm \mathrm{SD})$ & $157.5 \pm 18.7$ \\
\hline Clinic DBP $(\mathrm{mmHg} \pm \mathrm{SD})$ & $89.1 \pm 13.3$ \\
\hline Clinic pulse rate (beats/min $\pm \mathrm{SD}$ ) & $74.9 \pm 11.2$ \\
\hline Morning home SBP $(\mathrm{mmHg} \pm \mathrm{SD})$ & $156.9 \pm 16.4$ \\
\hline Morning home DBP (mmHg $\pm \mathrm{SD})$ & $89.7 \pm 12.0$ \\
\hline Morning home pulse rate (beats/min $\pm \mathrm{SD}$ ) & $72.7 \pm 10.7$ \\
\hline Evening home SBP $(\mathrm{mmHg} \pm \mathrm{SD})$ & $150.2 \pm 17.6$ \\
\hline Evening home DBP $(\mathrm{mmHg} \pm \mathrm{SD})$ & $85.6 \pm 12.2$ \\
\hline Evening home pulse rate (beats/min $\pm \mathrm{SD}$ ) & $72.5 \pm 9.6$ \\
\hline \multicolumn{2}{|l|}{ Patient classification ( $n[\%])$} \\
\hline Poorly controlled hypertension & $4,047[83.4]$ \\
\hline Masked hypertension & $478[9.9]$ \\
\hline White coat hypertension & $147[3.0]$ \\
\hline Well-controlled hypertension & $180[3.7]$ \\
\hline \multicolumn{2}{|l|}{ Time since diagnosis $(n[\%])$} \\
\hline$<1$ year & $1,146[23.6]$ \\
\hline 1 to $<5$ years & $980[20.2]$ \\
\hline 5 to $<10$ years & $398[8.2]$ \\
\hline$\geq 10$ years & $1,370[28.2]$ \\
\hline Unknown & $958[19.7]$ \\
\hline \multicolumn{2}{|l|}{ Comorbid conditions $(n[\%])$} \\
\hline Any & $3,208[66.1]$ \\
\hline Hyperlipidemia & $1,639[33.8]$ \\
\hline Diabetes mellitus & $864[17.8]$ \\
\hline Heart disease & $550[11.3]$ \\
\hline Hepatic disease & $366[7.5]$ \\
\hline Cerebrovascular disorder & $358[7.4]$ \\
\hline Gastrointestinal disorder & $355[7.3]$ \\
\hline Renal disease & $198[4.1]$ \\
\hline Respiratory disease & $169[3.5]$ \\
\hline
\end{tabular}


Table 1 continued

\begin{tabular}{lc}
\hline Characteristic & \multicolumn{1}{l}{ Value } \\
\hline Malignant neoplasm & $67[1.4]$ \\
Other & $851[17.5]$ \\
Previous treatment with antihypertensive drugs $(n[\%])$ & \\
Any & $2,650[54.6]$ \\
ARB & $1,775[36.6]$ \\
Calcium antagonist & $1,116[23.0]$ \\
$\beta$-blocker & $368[7.6]$ \\
ACE inhibitor & $322[6.6]$ \\
Diuretic & $289[6.0]$ \\
$\alpha$-Blocker & $182[3.8]$ \\
Other & $69[1.4]$ \\
Timing of home BP measurement $(n[\%])$ & \\
Before breakfast and before dosing & $4,132[85.2]$ \\
After breakfast and after dosing & $518[10.7]$ \\
Before breakfast and after dosing & $88[1.8]$ \\
After breakfast and before dosing & $99[2.0]$ \\
Not specified/unknown & $15[0.3]$ \\
\hline$A C E$. &
\end{tabular}

$A C E$ angiotensin converting enzyme, $A R B$ angiotensin receptor blocker, $B M I$ body mass index, $B P$ blood pressure, $D B P$ diastolic blood pressure, $S B P$ systolic blood pressure, $S D$ standard deviation

Table 2 Dosage of azelnidipine $(n=4,852)$

\begin{tabular}{lc}
\hline Parameter & Value \\
\hline Initial daily dose & \\
Mean \pm SD (mg) & $13.2 \pm 3.9$ \\
$\leq 4 \mathrm{mg}(n[\%])$ & $26[0.5]$ \\
$8 \mathrm{mg}(n[\%])$ & $1,661[34.2]$ \\
$16 \mathrm{mg}(n[\%])$ & $3,157[65.1]$ \\
$\geq 24 \mathrm{mg}(n[\%])$ & $8[0.2]$ \\
Maximal daily dose & $14.2 \pm 3.6$ \\
Mean \pm SD (mg) & $12[0.2]$ \\
$4 \mathrm{mg}(n[\%])$ & $1,136[23.4]$ \\
$8 \mathrm{mg}(n[\%])^{\mathrm{a}}$ & $3,681[75.9]$ \\
$16 \mathrm{mg}(n[\%])$ & $23[0.5]$ \\
$\geq 24 \mathrm{mg}(n[\%])$ & \\
\hline$S D$ standard deviation & \\
a Includes six patients who took $12 \mathrm{mg}$ &
\end{tabular}

dose and the maximal daily dose). Doses smaller or greater than the approved doses of 8-16 mg were hardly ever used. The mean initial daily dose was $13.2 \pm 3.9 \mathrm{mg}$, and the mean maximal daily dose was $14.2 \pm 3.6 \mathrm{mg}$.

Table 3 details the concomitant drugs used by patients at baseline. Antihypertensive drugs other than the study drug, antihyperlipidemic drugs, and antidiabetic drugs were concomitantly used in $45.5 \%, 20.1 \%$, and $10.6 \%$ of patients, respectively.
Table 3 Concomitant drugs used at baseline $(n=4,852)$

\begin{tabular}{lc}
\hline Concomitant drug & $n[\%]$ \\
\hline Any & $3,168[65.3]$ \\
Antihypertensive drugs & \\
Any & $2,210[45.5]$ \\
ARB & $1,743[35.9]$ \\
$\beta$-Blocker & $337[6.9]$ \\
Diuretic & $273[5.6]$ \\
ACE inhibitor & $261[5.4]$ \\
Calcium antagonist & $163[3.4]$ \\
$\alpha$-Blocker & $156[3.2]$ \\
Other & $61[1.3]$ \\
Antihyperlipidemic drug & $976[20.1]$ \\
Antidiabetic drug & $515[10.6]$ \\
Other & $1,747[36.0]$ \\
\hline ACE
\end{tabular}

$A C E$ angiotensin converting enzyme, $A R B$ angiotensin receptor blocker

\subsection{Blood Pressure and Pulse Rate-Lowering Effects}

Figure 2 and Table 4 show the changes in the mean SBP, DBP, and pulse rates at each timepoint. The clinic, morning home, and evening home measurements of SBP, DBP, and pulse rates decreased significantly by week 4 as compared with baseline $(p<0.0001)$, and these improvements were maintained at 16 weeks $(p<0.0001)$.

Table 5 shows the mean BP and pulse rate values before and after treatment with the study drug, and the changes in these. The mean changes in SBP/DBP were $-18.7 \pm$ $19.9 /-10.2 \pm 12.4 \mathrm{mmHg}$ (clinic), $-19.3 \pm 17.4 /-10.2 \pm$ $10.8 \mathrm{mmHg}$ (morning home), and $-16.9 \pm 17.0 /-9.4 \pm$ $10.6 \mathrm{mmHg}$ (evening home), and all changes were significant $(p<0.0001)$. The mean changes in pulse rates were $-3.5 \pm$ 9.5 beats/min (clinic), $-3.7 \pm 8.0$ beats/min (morning home), and $-3.5 \pm 7.3$ beats/min (evening home), and all reductions were significant $(p<0.0001)$.

Table 6 shows changes in patient classification based on both clinic SBP and morning home SBP measured before and after azelnidipine treatment. The proportion of patients with clinic SBP of $<140 \mathrm{mmHg}$ increased from $12.9 \%$ before azelnidipine administration to $56.1 \%$ after azelnidipine administration, and the proportion of patients with morning home SBP of $<135 \mathrm{mmHg}$ increased from $6.6 \%$ to $43.3 \%$. The patient classification, as determined by both clinic SBP and morning home SBP, improved significantly ( $p<0.0001$ according to the McNemar test).

Hypertension was deemed well-controlled in $32.2 \%$ of patients after administration of azelnidipine. Of the patients with poorly controlled or masked hypertension before azelnidipine treatment, $41.0 \%$ and $47.1 \%$, respectively, achieved morning home SBP of $<135 \mathrm{mmHg}$ by the 
Fig. 2 Changes in a clinic, morning home, and evening home blood pressure (BP) and b clinic, morning home, and evening home pulse rates after azelnidipine treatment. $* p<0.0001$ vs. baseline, according to Dunnett's test. $D B P$ diastolic blood pressure, $S B P$ systolic blood pressure
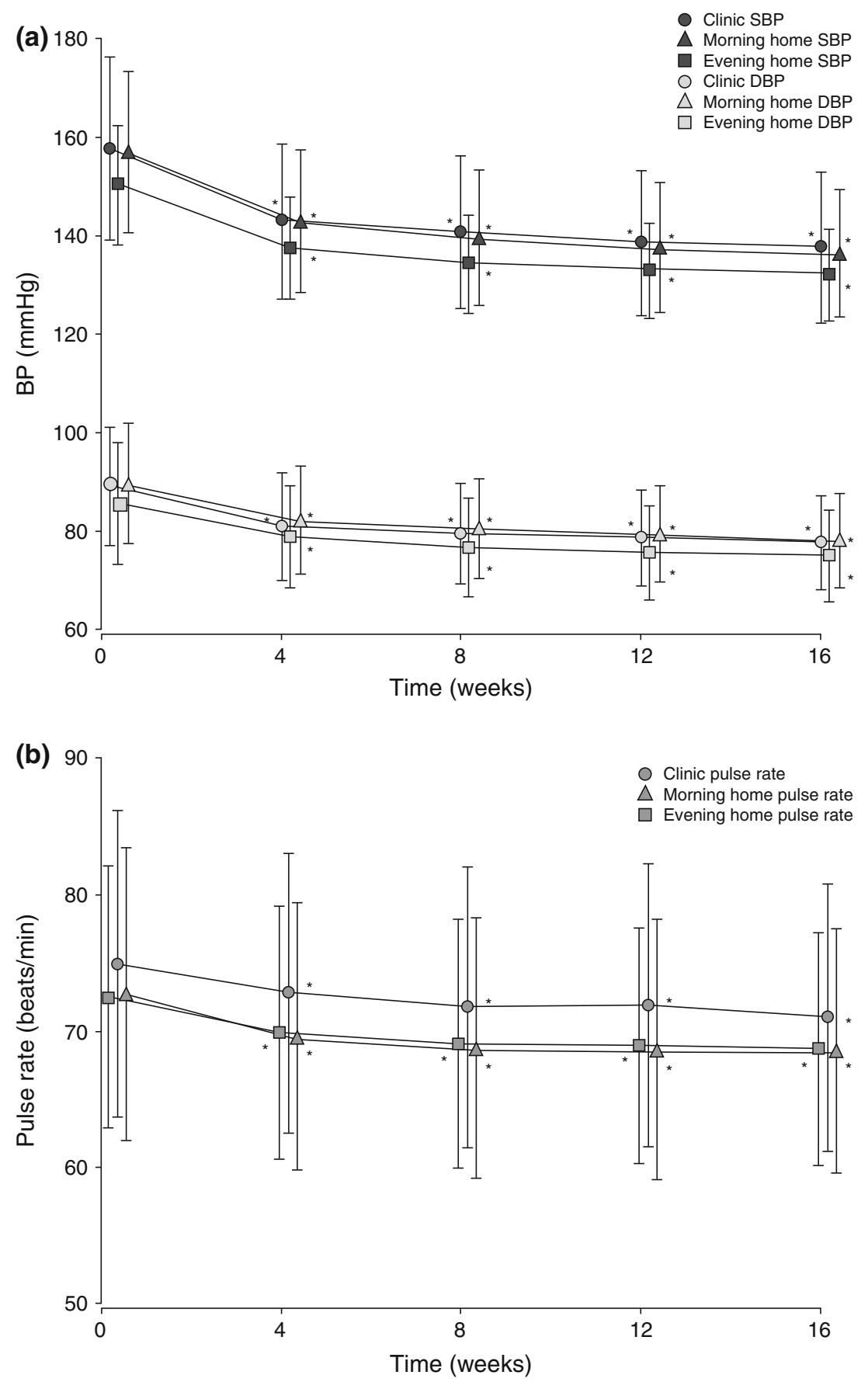

completion of the investigation, and $29.7 \%$ and $41.1 \%$, respectively, had well-controlled hypertension. Figure 3 shows a scatter diagram of the patients classified by clinic SBP and morning home SBP before and after azelnidipine treatment. Improvements in both clinic SBP and morning home SBP were evident after azelnidipine treatment. A similar analysis conducted in just those patients who complied with the study protocol yielded similar results.

\subsection{Safety}

Table 7 shows adverse drug reactions reported in the safety analysis population, classified according to their MedDRA Preferred Terms. Adverse drug reactions occurred in $2.92 \%$ of patients $(154 / 5,265)$, and the incidences of adverse drug reactions commonly associated with calcium antagonists were $0.42 \%$ for dizziness, $0.04 \%$ for 'dizziness postural', $0.32 \%$ for headache, $0.17 \%$ for hot flushes, 
Table 4 Time course of blood pressure and pulse rate changes

\begin{tabular}{|c|c|c|c|c|c|c|}
\hline \multicolumn{2}{|l|}{ Parameter } & \multirow[t]{2}{*}{ Baseline } & \multirow[t]{2}{*}{ Week 4} & \multirow[t]{2}{*}{ Week 8} & \multirow[t]{2}{*}{ Week 12} & \multirow[t]{2}{*}{ Week 16} \\
\hline \multicolumn{2}{|l|}{ Clinic } & & & & & \\
\hline \multirow[t]{2}{*}{ SBP } & $n$ & 4,852 & 3,300 & 3,011 & 2,854 & 3,295 \\
\hline & mmHg (mean \pm SD) & $157.5 \pm 18.7$ & $143.0 \pm 15.9$ & $140.9 \pm 15.7$ & $139.0 \pm 14.8$ & $138.3 \pm 15.1$ \\
\hline \multirow[t]{2}{*}{ DBP } & $n$ & 4,851 & 3,299 & 3,010 & 2,853 & 3,295 \\
\hline & mmHg (mean \pm SD) & $89.1 \pm 13.3$ & $81.1 \pm 11.3$ & $79.7 \pm 11.0$ & $79.1 \pm 10.7$ & $78.4 \pm 10.6$ \\
\hline \multirow[t]{2}{*}{ Pulse rate } & $n$ & 3,736 & 2,483 & 2,236 & 2,151 & 2,577 \\
\hline & beats/min (mean $\pm \mathrm{SD})$ & $74.9 \pm 11.2$ & $72.8 \pm 10.3$ & $71.8 \pm 10.3$ & $72.0 \pm 10.4$ & $71.1 \pm 9.8$ \\
\hline \multicolumn{7}{|c|}{ Morning home } \\
\hline \multirow[t]{2}{*}{ SBP } & $n$ & 4,852 & 3,138 & 2,796 & 2,835 & 3,281 \\
\hline & mmHg (mean \pm SD) & $156.9 \pm 16.4$ & $143.0 \pm 14.5$ & $140.0 \pm 13.9$ & $138.3 \pm 13.2$ & $137.1 \pm 12.9$ \\
\hline \multirow[t]{2}{*}{ DBP } & $n$ & 4,840 & 3,136 & 2,793 & 2,828 & 3,275 \\
\hline & mmHg (mean \pm SD) & $89.7 \pm 12.0$ & $82.4 \pm 11.0$ & $80.8 \pm 10.1$ & $79.8 \pm 9.8$ & $78.9 \pm 9.6$ \\
\hline \multirow[t]{2}{*}{ Pulse rate } & $n$ & 3,573 & 2,444 & 2,201 & 2,274 & 2,620 \\
\hline & beats/min $($ mean $\pm \mathrm{SD})$ & $72.7 \pm 10.7$ & $69.6 \pm 9.8$ & $68.8 \pm 9.5$ & $68.7 \pm 9.6$ & $68.7 \pm 9.0$ \\
\hline \multicolumn{7}{|c|}{ Evening home } \\
\hline \multirow[t]{2}{*}{ SBP } & $n$ & 2,546 & 1,869 & 1,689 & 1,738 & 1,940 \\
\hline & $\operatorname{mmHg}($ mean $\pm \mathrm{SD})$ & $150.2 \pm 17.6$ & $137.5 \pm 14.4$ & $134.5 \pm 13.2$ & $133.5 \pm 13.1$ & $132.7 \pm 12.8$ \\
\hline \multirow[t]{2}{*}{$\mathrm{DBP}$} & $n$ & 2,543 & 1,869 & 1,689 & 1,736 & 1,940 \\
\hline & $\operatorname{mmHg}($ mean $\pm \mathrm{SD})$ & $85.6 \pm 12.2$ & $78.8 \pm 10.4$ & $76.9 \pm 9.9$ & $76.0 \pm 9.5$ & $75.8 \pm 9.3$ \\
\hline \multirow[t]{2}{*}{ Pulse rate } & $n$ & 2,191 & 1,614 & 1,476 & 1,548 & 1,734 \\
\hline & beats/min $($ mean $\pm \mathrm{SD})$ & $72.5 \pm 9.6$ & $69.9 \pm 9.3$ & $69.1 \pm 9.1$ & $69.0 \pm 8.7$ & $68.8 \pm 8.6$ \\
\hline
\end{tabular}

$D B P$ diastolic blood pressure, $S B P$ systolic blood pressure, $S D$ standard deviation

Table 5 Clinical improvement from baseline

\begin{tabular}{|c|c|c|c|c|c|}
\hline Parameter & & Baseline & Endpoint & Endpoint minus baseline & $p$ value \\
\hline \multicolumn{6}{|l|}{ Clinic } \\
\hline \multirow[t]{2}{*}{ SBP } & $n$ & 4,852 & 4,512 & 4,512 & \\
\hline & mmHg (mean $\pm \mathrm{SD})$ & $157.5 \pm 18.7$ & $138.9 \pm 15.5$ & $-18.7 \pm 19.9$ & $<0.0001$ \\
\hline \multirow[t]{2}{*}{ DBP } & $n$ & 4,851 & 4,511 & 4,511 & \\
\hline & mmHg $($ mean \pm SD) & $89.1 \pm 13.3$ & $78.9 \pm 10.8$ & $-10.2 \pm 12.4$ & $<0.0001$ \\
\hline \multirow[t]{2}{*}{ Pulse rate } & $n$ & 3,736 & 3,487 & 3,340 & \\
\hline & beats/min $($ mean $\pm \mathrm{SD})$ & $74.9 \pm 11.2$ & $71.5 \pm 10.1$ & $-3.5 \pm 9.5$ & $<0.0001$ \\
\hline \multicolumn{6}{|c|}{ Morning home } \\
\hline \multirow[t]{2}{*}{ SBP } & $n$ & 4,852 & 4,200 & 4,200 & \\
\hline & mmHg (mean $\pm \mathrm{SD})$ & $156.9 \pm 16.4$ & $137.7 \pm 13.3$ & $-19.3 \pm 17.4$ & $<0.0001$ \\
\hline \multirow[t]{2}{*}{ DBP } & $n$ & 4,840 & 4,190 & 4,187 & \\
\hline & mmHg $($ mean $\pm \mathrm{SD})$ & $89.7 \pm 12.0$ & $79.4 \pm 9.7$ & $-10.2 \pm 10.8$ & $<0.0001$ \\
\hline \multirow[t]{2}{*}{ Pulse rate } & $n$ & 3,573 & 3,275 & 3,076 & \\
\hline & beats/min $($ mean $\pm \mathrm{SD})$ & $72.7 \pm 10.7$ & $68.9 \pm 9.3$ & $-3.7 \pm 8.0$ & $<0.0001$ \\
\hline \multicolumn{6}{|c|}{ Evening home } \\
\hline \multirow[t]{2}{*}{ SBP } & $n$ & 2,546 & 2,418 & 2,108 & \\
\hline & mmHg $($ mean $\pm \mathrm{SD})$ & $150.2 \pm 17.6$ & $133.0 \pm 13.1$ & $-16.9 \pm 17.0$ & $<0.0001$ \\
\hline \multirow[t]{2}{*}{ DBP } & $n$ & 2,543 & 2,416 & 2,105 & \\
\hline & $\mathrm{mmHg}($ mean $\pm \mathrm{SD})$ & $85.6 \pm 12.2$ & $76.0 \pm 9.4$ & $-9.4 \pm 10.6$ & $<0.0001$ \\
\hline \multirow[t]{2}{*}{ Pulse rate } & $n$ & 2,191 & 2,127 & 1,833 & \\
\hline & beats/min (mean $\pm \mathrm{SD})$ & $72.5 \pm 9.6$ & $69.0 \pm 8.7$ & $-3.5 \pm 7.3$ & $<0.0001$ \\
\hline
\end{tabular}

$D B P$ diastolic blood pressure, $S B P$ systolic blood pressure, $S D$ standard deviation

a Significance of changes from baseline, according to paired $t$-test 
Table 6 Patient classification based on morning home systolic blood pressure (SBP) and clinic SBP before and after azelnidipine treatment $(n=4,074)$

\begin{tabular}{|c|c|c|c|c|c|}
\hline \multirow[t]{2}{*}{ Parameter at baseline } & \multicolumn{5}{|c|}{ Endpoint $(n[\%])^{\mathrm{a}}$} \\
\hline & $\begin{array}{l}\text { Well-controlled } \\
\text { hypertension }\end{array}$ & $\begin{array}{l}\text { White coat } \\
\text { hypertension }\end{array}$ & $\begin{array}{l}\text { Poorly controlled } \\
\text { hypertension }\end{array}$ & $\begin{array}{l}\text { Masked } \\
\text { hypertension }\end{array}$ & Total \\
\hline Well-controlled hypertension & 84 [58.3] & 16 [11.1] & $21[14.6]$ & $23[16.0]$ & $144[3.5]$ \\
\hline White coat hypertension & 54 [42.9] & $28[22.2]$ & $30[23.8]$ & $14[11.1]$ & $126[3.1]$ \\
\hline Poorly controlled hypertension & $1,016[29.7]$ & $386[11.3]$ & $1,219[35.6]$ & $799[23.4]$ & $3,420[83.9]$ \\
\hline Masked hypertension & 158 [41.1] & $23[6.0]$ & 67 [17.4] & $136[35.4]$ & $384[9.4]$ \\
\hline Total & 1,312 [32.2] & $453[11.1]$ & 1,337 [32.8] & 972 [23.9] & 4,074 [100.c \\
\hline
\end{tabular}

a The proportions were calculated using the baseline data as denominators

Fig. 3 Changes in patient classification according to morning home and clinic systolic blood pressure (SBP) [n=4,074]: a classification before azelnidipine treatment; b classification at the study endpoint (a)

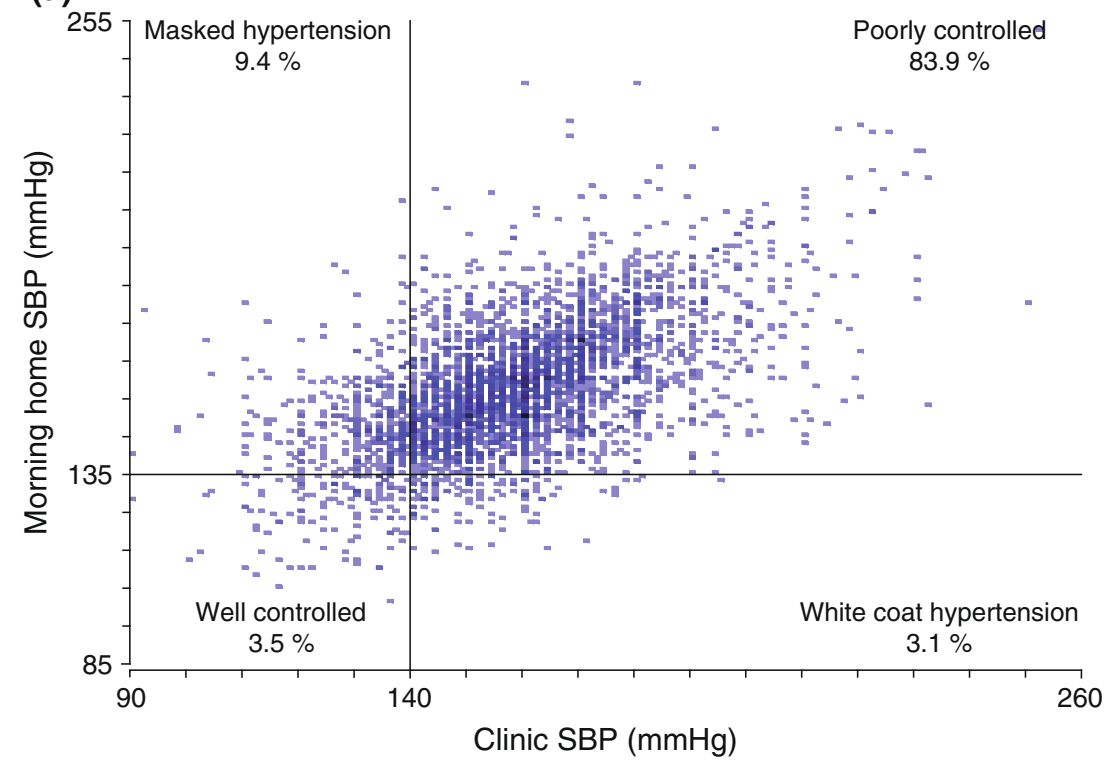

(b)

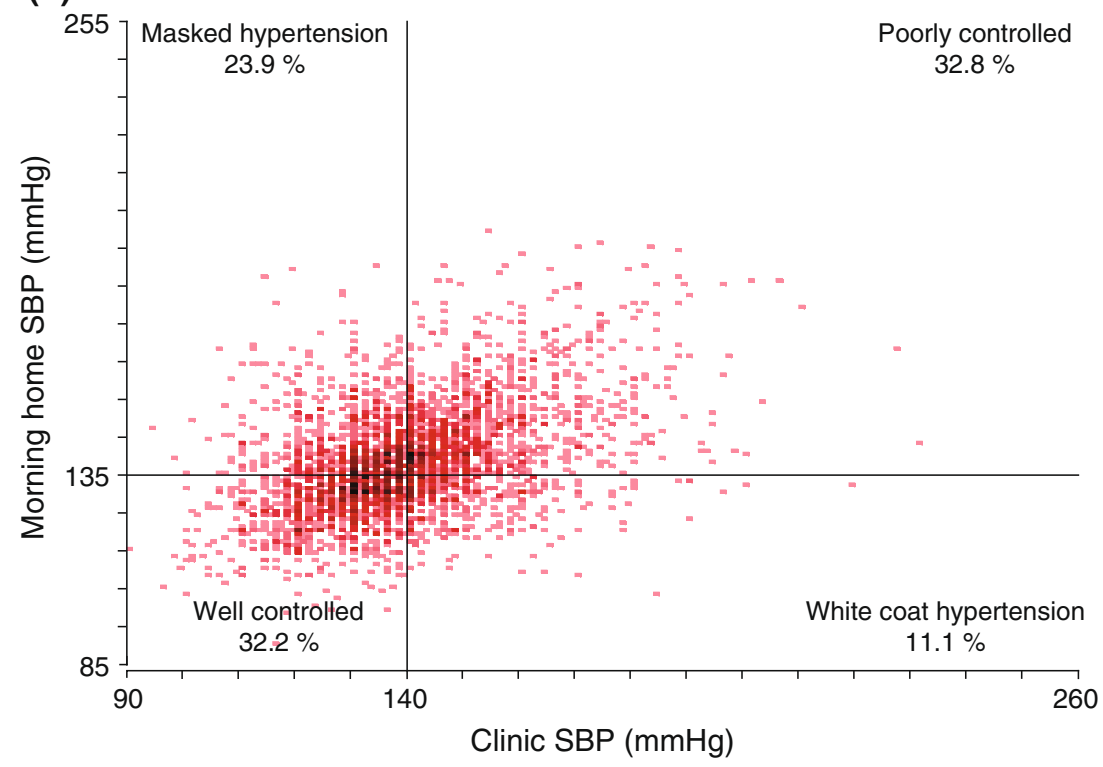


Table 7 Incidence of adverse drug reactions (ADRs) reported in the safety analysis population $(n=5,265)$

\begin{tabular}{lr}
\hline Parameter & $n[\%]$ \\
\hline No. of patients who developed an ADR & $154[2.92]$ \\
Total no. of ADRs ${ }^{\mathrm{a}}$ & 193 \\
No. of ADRs ${ }^{\mathrm{a}}$ commonly associated $^{\text {with calcium antagonists }}$ & 63 \\
Dizziness & $22[0.42]$ \\
Headache & $17[0.32]$ \\
Hot flushes & $9[0.17]$ \\
Palpitations & $6[0.11]$ \\
Edema peripheral & $5[0.09]$ \\
Dizziness postural & $2[0.04]$ \\
Edema & $2[0.04]$ \\
\hline
\end{tabular}

a These ADRs are classified according to their Medical Dictionary for Regulatory Activities (MedDRA) Preferred Terms

$0.11 \%$ for palpitations, $0.04 \%$ for edema, and $0.09 \%$ for 'edema peripheral'.

\section{Discussion}

Home BP is reported to be a better predictor of survival outcome than clinic BP $[3,11]$. It is very important for treatment of hypertension to accurately diagnose and control morning hypertension, which carries a serious risk of cardiovascular and target organ disorders. However, morning home BP was controlled in only $39 \%$ of patients who were taking antihypertensive drug treatment in the J-MORE Study. Masked hypertension was reported in more than half of the patients whose clinic BP was controlled by antihypertensive drugs (Fig. 4) [2]. We investigated the suppressive effect of azelnidipine on clinic BP, morning home $\mathrm{BP}$, and morning hypertension, using data collected in the At-HOME Study. The effect of azelnidipine on pulse rates was also examined.

Clinic, morning home, and evening home SBP and DBP were significantly lowered by week $4(p<0.0001)$, and treatment had a significant BP-lowering effect $(p<0.0001)$ throughout the 16-week treatment period. Moreover, the changes in clinic BP, morning home BP, and evening home BP were significant $(p<0.0001)$. A greater proportion of patients achieved clinic SBP of $<140 \mathrm{mmHg}$ (56.1\%) and morning home SBP of $<135 \mathrm{mmHg}(43.3 \%)$ by week 16 in the present study than in the J-MORE Study (44\% for clinic SBP and $39 \%$ for morning home SBP), and a greater proportion of patients achieved well-controlled hypertension (as assessed by both clinic SBP and morning SBP) in the present study than in the J-MORE Study $(32.2 \%$ vs. $21 \%)$. The clinical effects of

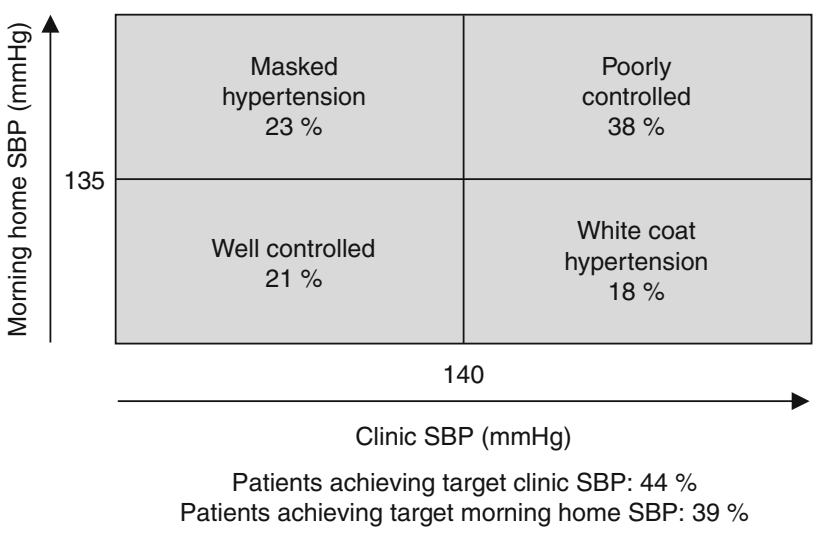

Fig. 4 Patient classification according to clinic systolic blood pressure (SBP) and morning home SBP in the Jichi MorningHypertension Research (J-MORE) Study [2]

azelnidipine were assumed to be superior to those of conventional antihypertensive therapy (mainly calcium antagonists). In $41.0 \%$ of patients with poorly controlled hypertension and $47.1 \%$ of patients with masked hypertension at baseline, morning home BP was well controlled by azelnidipine treatment. Ohkubo et al. [12] and Kario et al. [13] reported that morning hypertension increased cerebrovascular and cardiovascular disease and stroke risks, and predicted asymptomatic cerebral infarction in the elderly [1]. The Japan Morning Surge-1 (JMS-1) Study reported that strict control of morning hypertension could suppress hypertension-related organ damage [14]. When morning home BP is not measured in hypertensive patients, treatment of morning hypertension is likely to be inefficient, so measurement and strict control of morning home $\mathrm{BP}$ are extremely important. Azelnidipine is a slow-acting, sustained-effect dihydropyridine calcium antagonist and an antihypertensive drug that can be administered once daily [15]. Because it has greater higher lipophilicity than other calcium antagonists, it has superior affinity for vascular tissues and prolonged distribution in them; strong binding to L-type calcium channels by the 'membrane approach'; and slow, sustained, and strong hypotensive and anti-atherosclerotic activities [16, 17]. The results of this study suggest that azelnidipine has a sustained BP-lowering effect and usefulness in patients with morning hypertension at high risk of cardiovascular disease.

Clinic, morning home, and evening home measurements showed a significant decrease in pulse rates $(p<0.0001)$ starting at week 4 and continuing up to week 16 $(p<0.0001)$, and the changes from baseline to the study endpoint were sustained $(p<0.0001)$. The BP-lowering effect of azelnidipine is reported to be slower than that of amlodipine - a widely used conventional drug, which is 
reported to have a slow BP-lowering effect and is therefore less likely to be affected by baroreceptor reflex-mediated changes in sympathetic nervous system activity. Since addition of a high-concentration product does not reduce myocardial contraction, azelnidipine only mildly reduces the pulse rate rather than increasing it [18]. In the Framingham Study report, an increase in pulse rates was related to an increase in the rate of cardiovascular disease events over a long period [19]. Many calcium antagonists increase pulse rates by activating the sympathetic nervous system via the baroreceptor reflex $[20,21]$. Other dihydropyridine calcium antagonists do not have the distinct pulse rate-lowering effect of azelnidipine, and thus azelnidipine is considered one of the most important (and is one of the most frequently used) calcium antagonists available to improve the prognosis of hypertensive patients who require long-term treatment.

The incidence of adverse drug reactions was lower in this investigation than in an earlier 'Drug Use Results Survey' of azelnidipine [22] (2.92 \% vs. $3.5 \%)$. The incidence of adverse drug reactions often observed with the dihydropyridine calcium antagonist was low in the current study: $0.42 \%$ for dizziness, $0.32 \%$ for headache, $0.17 \%$ for hot flushes, $0.11 \%$ for palpitations, $0.09 \%$ for edema peripheral, $0.04 \%$ for dizziness postural, and $0.04 \%$ for edema.

The results of this investigation were considered to reflect actual routine hypertension treatment. Under conditions where strict $\mathrm{BP}$ control is required in hypertensive patients [23, 24], measurement of morning home BP is very important for diagnosing and treating morning hypertension and for improving patient compliance. Azelnidipine is also considered one of the most useful antihypertensive drugs for its sustained BP-lowering effect and its pulse rate-lowering effect.

\section{Conclusion}

The At-HOME Study of azelnidipine tablets administered over a 16-week standard observation period was performed between May 2006 and September 2007. The results were reviewed in order to evaluate the drug's effects on clinic and home BP, morning hypertension, and pulse rates. The following results were obtained in 5,433 patients who were registered by the central registration method from 1,011 medical institutions across Japan:

1 After azelnidipine treatment, clinic, morning home, and evening home BP measurements showed significant lowering of SBP and DBP by week 4 and persistence of the effect up to week 16. The mean SBP/DBP changes from baseline were $-18.7 \pm 19.9 /-10.2 \pm 12.4 \mathrm{mmHg}$ (clinic), $-19.3 \pm 17.4 /-10.2 \pm 10.8 \mathrm{mmHg}$ (morning home), and $-16.9 \pm 17.0 /-9.4 \pm 10.6 \mathrm{mmHg}$ (evening home), and all improvements were significant.

2 Clinic SBP of $<140 \mathrm{mmHg}$ was achieved in $56.1 \%$ of patients after azelnidipine treatment, and morning home SBP of $<135 \mathrm{mmHg}$ was achieved in $43.3 \%$ of patients. These results are better than those of the J-MORE Study. Hypertensive patients with well-controlled hypertension after azelnidipine treatment constituted $32.2 \%$ of the entire study population. Of the patients with poorly controlled or masked hypertension before azelnidipine treatment, $41.0 \%$ and $47.1 \%$, respectively, achieved morning home SBP of $<135 \mathrm{mmHg}$.

3 After azelnidipine treatment, pulse rates were significantly lowered by week 4 , and the effects persisted up to week 16. The mean changes from baseline were $-3.5 \pm 9.5$ beats/min (clinic), $-3.7 \pm 8.0$ beats $/ \mathrm{min}$ (morning home), and $-3.5 \pm 7.3$ beats/min (evening home), and these significant reductions persisted throughout the period of observation.

4 The incidence of adverse drug reactions was low at $2.92 \%$, with reactions occurring in $154 / 5,265$ patients.

On the basis of these results, the authors consider azelnidipine to be one of the most useful antihypertensive drugs because of its reliable and persistent BP-lowering effects, in addition to its pulse rate-lowering effect.

Acknowledgments The authors would like to thank all of the investigators who cooperated with the At-HOME Study and provided valuable data. The authors would also like to thank Rod McNab and Nila Bhana from inScience Communications, Springer Healthcare (Auckland, New Zealand), who provided English-language editing. This assistance, as well as the translation from Japanese to English, was funded by Daiichi Sankyo Co., Ltd (Tokyo, Japan). Kazuyuki Shimada is now employed by Oyama Municipal Hospital (Tochigi, Japan). Masahiro Komiya is now employed by Daiichi Sankyo Healthcare Co., Ltd (Tokyo, Japan). The authors have no other conflicts of interest that are directly relevant to the content of this article. A version of this manuscript was previously published in Japanese in the Journal of Clinical Therapeutics \& Medicine [2008;24(12): 1083-98]. The publisher of the Journal of Clinical Therapeutics \& Medicine has given permission for publication of this article in English.

Open Access This article is distributed under the terms of the Creative Commons Attribution Noncommercial License which permits any noncommercial use, distribution, and reproduction in any medium, provided the original author(s) and the source are credited.

\section{References}

1. Kario K, Pickering TG, Umeda Y, et al. Morning surge in blood pressure as a predictor of silent and clinical cerebrovascular disease in elderly hypertensives: a prospective study. Circulation. 2003;107(10):1401-6. 
2. Kario K, Eguchi K, Umeda Y, et al. Morning surge in blood pressure as a predictor of silent and clinical cerebrovascular disease in elderly hypertension. Circulation. 2003;108(10):72e-3e.

3. Pickering TG, Davidson K, Gerin W, et al. Masked hypertension. Hypertension. 2002;40(6):795-6.

4. Okubo T, Imai Y, Tsuji I, et al. Home blood pressure measurement has a stronger predictive power for mortality than does screening blood pressure measurement: a population-based observation in Ohasama, Japan. J Hypertens. 1998;16:971-5.

5. Ménard J, Chatellier G, Day M, et al. Self-measurement of blood pressure at home to evaluate drug effects by the trough:peak ratio. J Hypertens. 1994;12(Suppl 8):S21-5.

6. Oizumi K, Nishino H, Koike H, et al. Antihypertensive effects of CS-905, a novel dihydropyridine $\mathrm{Ca}^{++}$channel blocker, in SHR [in Japanese]. Jpn J Pharmacol. 1989;51:57-64.

7. Oizumi K, Nishino H, Miyamoto M, et al. Beneficial renal effects of CS-905, a novel dihydropyridine calcium blocker, in SHR [in Japanese]. Jpn J Pharmacol. 1989;51(4):501-8.

8. Ikeda K, Nishino H, Oizumi K, et al. Antihypertensive effects of CS-905, a new calcium antagonist in cholesterol-fed rabbits [in Japanese]. Jpn J Pharmacol. 1992;58(Suppl):342.

9. Kuramoto K, Ichikawa S, Hirai A, et al. Azelnidipine and amlodipine: a comparison of their pharmacokinetics and effects on ambulatory blood pressure. Hypertens Res. 2003;26:201-8.

10. Kumagaya $H$, Onami $T$, Iigatani $Y$, et al. Mechanism of a reduction in heart rate by azelnidipine as investigated in terms of the peripheral and central nervous systems [in Japanese]. Prog Med (Jpn). 2004;24(11):2659-64.

11. Sega R, Facchetti R, Bombelli M, et al. Prognostic value of ambulatory and home blood pressure compared with office blood pressure in the general population: follow-up results from the Pressioni Arteriose Monitorate e Loro Associazioni (PAMELA) Study. Circulation. 2005;111:1777-83.

12. Ohkubo T, Kikuya M, Metoki H, et al. Prognosis of "masked" hypertension and "white-coat" hypertension detected by 24-h ambulatory blood pressure monitoring 10-year follow-up from the Ohasama study. J Am Coll Cardiol. 2005;46(3):508-15.

13. Kario K, Ishikawa J, Pickering TG, et al. Morning hypertension: the strongest independent risk factor for stroke in elderly hypertensive patients. Hypertens Res. 2006;29(8):581-7.

14. Kario K, Matsui Y, Shibasaki S, et al. An alpha-adrenergic blocker titrated by self-measured blood pressure and microalbuminuria in patients with morning hypertension: the Japan Morning Surge-1 Study. J Hypertens. 2008;26(6):1257-65.

15. Yamamoto Y, Sonoyama K, Matsubara K, et al. The status of hypertension management in Japan in 2000. Hypertens Res. 2002;25(5):717-25.

16. Sada T, Mizuno M, Miyama T, et al. Pharmacological characteristics of azelnidipine, a long-acting calcium antagonist, having vascular affinity (No. 2) - antihypertensive effect and pharmacokinetics in spontaneously hypertensive rats (SHR) [in Japanese]. Jpn Pharmacol Ther. 2002;30(9):711-20.

17. Sada T, Mizuno M, Oohata K, et al. Antiatherosclerotic effect of azelnidipine, a long-acting calcium antagonist with high lipophilicity, in cholesterol-fed rabbits [in Japanese]. Jpn Pharmacol Ther. 2002;30(9):721-8.

18. Yorikane R. Unique cardiac effect of azelnidipine, a novel calcium antagonist [in Japanese]. Bio Clin. 2003;18(13):1210-5.

19. Palatini $P$, Benetos A, Julius S. Impact of increased heart rate on clinical outcomes in hypertension: implications for antihypertensive drug therapy. Drugs. 2006;66(2):133-44.

20. Okabayashi J, Matsubayashi K, Sato T, et al. Effects of nifedipine and enalapril on the central nervous system in elderly hypertensive patients: power spectral analysis of heart rate variability [in Japanese]. Jpn J Geriatr. 1994;31(4):285-92.

21. Eguchi K, Kario K, Shimada K. Differential effects of a longacting angiotensin converting enzyme inhibitor (temocapril) and a long-acting calcium antagonist (amlodipine) on ventricular ectopic beats in older hypertensive patients. Hypertens Res. 2002;25(3):329-33.

22. Kitai T, Yoshida Y, Kuramoto K, et al. Use-results survey of azelnidipine $\left(\right.$ Calblock $\left.^{\circledR}\right)$ tablet [in Japanese]. J Clin Ther Med. 2005;21:511-27.

23. UK Prospective Diabetes Study Group. Efficacy of atenolol and captopril in reducing risk of macrovascular and microvascular complications in type 2 diabetes: UKPDS 39. BMJ. 1998;317 (7160):713-20.

24. Nippon Data 80 Research Group. Impact of elevated blood pressure on mortality from all causes, cardiovascular diseases, heart disease and stroke among Japanese: 14 year follow-up of randomly selected population from Japanese-Nippon data 80 . J Hum Hypertens. 2003;17(12):851-7. 